\title{
Nanoporous silica nanoparticles with spherical and anisotropic shape as fillers in dental composite materials
}

\begin{abstract}
The objective of this study was to test whether nanoporous silica nanoparticles can be employed as fillers in dental composite materials to improve their mechanical properties. These nanoporous silica nanoparticles were synthesized using sol-gel methods, in part modified by silanization, and thoroughly characterized. The nanoporous nanoparticles were added to dental resins to form nanocomposites (resins impregnated with nanoparticles) and hybrid composites (containing in addition conventional microfillers). The incorporation of these nanoporous nanoparticles in dental resins or composites was characterized by investigation of the complex viscosity and double bond conversion as well as by determination of flexural strength and Young's modulus. The dispersion of the nanofillers was examined by SEM and EDX imaging of fracture surfaces. Incorporation of small contents (1-3 wt \%) of unmodified nanoporous particles leads to improved mechanical properties. However, the incorporation of larger contents results in particle agglomeration and declining mechanical properties. This effect is less pronounced when the surface of the particles is modified with methacrylate residues, resulting in a lower agglomeration tendency and a more homogeneous filler dispersion. Surface properties and, concomitantly, dispersibility of the nanoparticles have a strong influence on mechanical properties. But the incorporation of nanoporous instead of solid nanoparticles
\end{abstract}

aThese two authors contributed equally to this work.

*Corresponding author: Henning Menzel, Technische Universität Braunschweig, Institut für Technische Chemie, Hans-SommerStraße 10, 38106 Braunschweig, Phone: +49 531391 5361, Fax:+49531391 5357, E-mail: h.menzel@tu-bs.de

Nico Timpe: Institut für Technische Chemie, Technische Universität Braunschweig, 38106 Braunschweig, Germany Hendrik Fullriede and Peter Behrens: Institut für Anorganische Chemie, Leibniz Universität Hannover, 30167 Hannover, Germany Lothar Borchers and Meike Stiesch: Klinik für Zahnärztliche Prothetik und Biomedizinische Werkstoffkunde, Medizinische Hochschule Hannover, 30625 Hannover, Germany into dental composite materials is indeed a possibility to improve the mechanical behavior. However, modification of the surface is necessary and the key to achieving uniform dispersion and, thereby, improving mechanical properties.

Keywords: anisotropic shape; hybrid composite; mechanical properties; nanoparticles; nanocomposite; nanoporosity.

DOI 10.1515/bnm-2014-0010

Received September 23, 2014; accepted November 7, 2014

\section{Introduction}

Composites belong to the most important materials in dentistry, and nowadays, the often used amalgam fillings are replaced by composite fillings as the restorative material of choice [1]. In addition to being less frequently subject to safety concerns, dental composite materials exhibit better esthetic appearance and steadily improving mechanical properties. Anterior lesions as well as smaller or mediumsized defects in the posterior region of the tooth can be restored by direct composite fillings. Composites are also used for prosthodontic treatments with crowns or fixed partial dentures as well as with removable dentures and offer many tooth substance saving and cost-effective treatment approaches [2].

Dental composite materials consist of inorganic fillers dispersed in an organic polymer matrix. The monomer 2,2-bis-[4-(methacryloxypropoxy)-phenyl]-propane (BisGMA) as photo-polymerizable dimethacrylate is most frequently used in restorative dentistry [3]. Due to its high viscosity, tri(ethylene glycol) dimethacrylate (TEGDMA) is usually added as diluent to improve handling properties. 
Microfillers as well as nanofillers are used. Common microfillers are ceramics in the form of ground glass splinters with an average size of $0.2-5.0 \mu \mathrm{m}$ which adjust color and opaqueness of the composite. Typical nanofillers are pyrogenic silica nanoparticles of spherical shape with a particle size of 10-100 nm, which are employed to improve the mechanical properties like hardness, flexural strength, and abrasion resistance [3].

One of the main disadvantages of dental composite resins is the still limited durability compared to amalgam fillings [4]. The reinforcement of dental composites by nanoparticles as fillers is crucial for the improvement of mechanical properties including enhanced durability. There exist investigations indicating that the release of spherical filler particles from the composite surface due to wear might result in loss of flexural strength [5]. A new approach to reinforce dental composite materials is the application of fiber-shaped nanofillers. Zhang et al. used naturally occurring crystalline silicate fibers as fillers and were able to improve the mechanical properties of the composite materials at low filler contents of $7.5 \mathrm{wt} \%$ or less $[6,7]$. These enhancements are ascribed to the socalled "bridging" effect: When a micro-crack is initiated within the composite by wear or stress, the inorganic fiber remains intact across the crack edges. The crack is stabilized and further opening is inhibited. The filler particles need to possess high aspect ratios and strength values for being able to act as bridging elements $[8,9]$. The crystalline silicate fibers used by Zhang et al. [6, 7], however, may not be biocompatible. They have a structure resembling that of asbestos and are suspicious of causing lung diseases or hemolysis [10, 11]. Thus we decided to investigate fibers of amorphous silica. In contrast to crystalline silicates, amorphous silica usually shows good biocompatibility and is approved by the U.S. Food and Drug Administration as GRAS (generally recognized as safe, GRN No. 321). Silica nanoparticles can be obtained via the sol-gel route and can be prepared with adjustable morphology and size. The silica nanoparticles typically used in dental materials are manufactured by flame pyrolysis and, therefore, are nonporous. The sol-gel route, however, by using structure-directing agents during the synthesis, offers the possibility to generate ordered pore systems [12]. These increase surface area for contact with the polymer matrix and could be used to build up drug-delivery systems [13].

Silica nanoparticles exhibit two kinds of surface groups: hydrophilic silanol groups and hydrophobic siloxane groups. On the particle surface of pyrogenic silica, a high content of hydrophobic siloxane groups can be found due to the high-temperature process of its preparation. In comparison, silica nanoparticles prepared at low temperatures from solution via the sol-gel route show a large quantity of silanol surface groups. The incorporation of such hydrophilic fillers into the hydrophobic monomer mixture during the composite preparation is difficult and typically leads to agglomeration of the nanoparticles so that they cannot fully exert their strengthening effects [14]. One way to prevent this is to modify the particle surface with hydrophobic entities. This can be performed using organosilanes in post-synthesis grafting procedures [15]. When these hydrophobic entities are organic methacrylate groups, they can be directly linked to the methacrylate derivative monomers during the photo-polymerization process and, this way, connect the inorganic filler particles with the organic matrix. This should lead to improved mechanical properties.

In this work, two different kinds of composite materials were evaluated. The first one consisted of resins containing both nano- and microparticles as fillers, thus resembling actually used commercial dental composites. It was prepared with a combination of a nanofiller and a dental glass filler at a total content of $50 \mathrm{wt} \%$. This type of material will subsequently be termed "hybrid composites". Additionally, a second composite material containing only small amounts of nanoparticles (0-10 wt $\%)$ was investigated to exclude the influence of the microfillers. These so-called "nanocomposites" offer a simplified model system for investigating only the effects of the nanoparticles on the mechanical properties of the composite materials. For the purpose of comparison, hybrid composites and nanocomposites were prepared using four types of nanofillers: commercial spherical nanofiller Aerosil OX 50, unmodified nanoporous anisotropic silica nanoparticles, modified nanoporous anisotropic silica nanoparticles, and modified nanoporous spherical silica nanoparticles.

\section{Results and discussion}

\section{Synthesis and characterization of aniso- tropic nanoporous silica nanoparticles}

As regards size and morphology of the synthesized silica fillers, the SEM image in Figure 1A shows non-aggregated spherical nanoparticles with a mean diameter of $30 \mathrm{~nm}$ and a narrow particle size distribution. The SEM images of the anisotropic silica particles reveal a fiber-like morphology (Figure 1B). These particles are mostly straight but some are also slightly curved. They show a broader distribution of length and morphology than the spherical particles but the diameters appear to be similar for all particles 

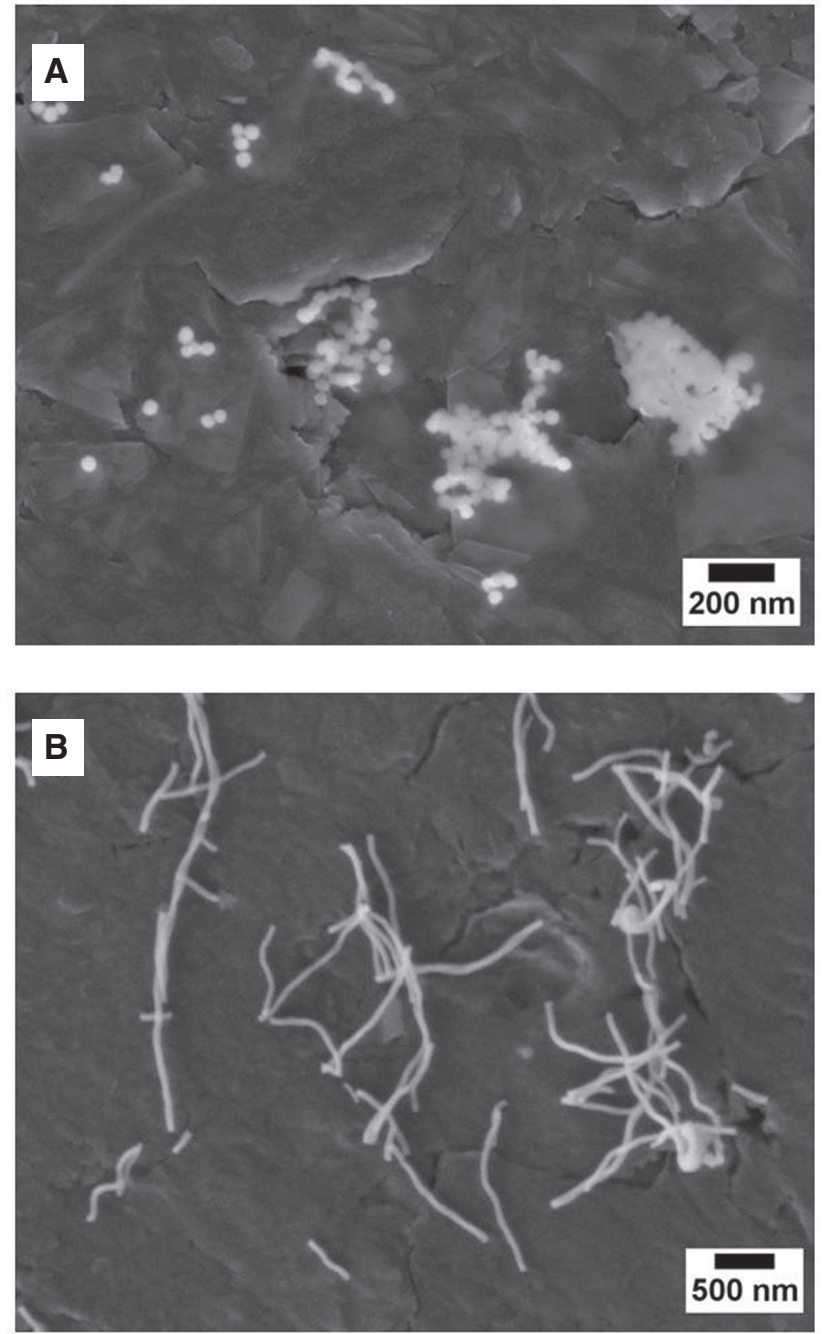

Figure 1 SEM images of spherical (A) and anisotropic (B) nanoporous silica nanoparticles.

at approximately $100 \mathrm{~nm}$. With lengths of several micrometers, these particles have high aspect ratios which should promote good bridging effects in composite materials.

The argon sorption measurements for investigation of the pore system of the filler particles resulted in adsorption and desorption isotherms as shown in the supporting information. They correspond to IUPAC-type IV isotherms [16] with more or less defined hysteresis trends. The anisotropic silica particles and the spherical particles exhibit surface areas of $980 \mathrm{~m}^{2} \mathrm{~g}^{-1}$ and $730 \mathrm{~m}^{2} \mathrm{~g}^{-1}$, respectively. In comparison, the surface area of the reference filler Aerosil OX 50 was determined to be $40 \mathrm{~m}^{2} \mathrm{~g}^{-1}$. The anisotropic silica nanoparticles exhibit a very narrow pore size distribution with an average pore width of $2.9 \mathrm{~nm}$, the spherical silica nanoparticles show an average pore diameter of $3.5 \mathrm{~nm}$. Thus, both types of particles are nanoporous.

\section{Modification of nanoporous silica nanoparticles}

After silanization reaction with MAPTMS for modification of surface properties, the IR spectra (Figure 2) of the modified anisotropic silica particles show additional absorption bands which can be ascribed to the $\alpha, \beta$-unsaturated methacrylate ester $\left(\mathrm{C}=\mathrm{O}\right.$ vibration at $1709 \mathrm{~cm}^{-1}$ and $\mathrm{C}-\mathrm{O}$ vibration at $\left.1317 \mathrm{~cm}^{-1}\right)$ and the propyl group (C-H vibrations at $2939 \mathrm{~cm}^{-1}$ and $1458 \mathrm{~cm}^{-1}$ ) [17]. In addition, the absorbance band at $3740 \mathrm{~cm}^{-1}$ disappears after the modification. This band can be assigned to free silanol groups on the silica surface [18] which have reacted with the modifying agent. Hence, the changes in the IR spectra indicate a successful modification of the silica particles with methacryloxy groups. Similar results were obtained for the spherical silica nanoparticles.

\section{Viscosity}

Viscosity is an important parameter in terms of handling dental composite pastes. Furthermore, viscosity influences the mobility of methacrylate groups and might affect the cross-linking reaction and double bond conversion. The viscosity of uncured composites is strongly influenced by the type of filler. Strongly aggregating fillers as the non-modified silica particles significantly increase the viscosity. A way to keep the viscosity on an acceptable level is the modification of the hydrophilic filler particles with hydrophobic silanes [19].

Figure 3A shows the complex viscosities of uncured nanocomposites for different types of nanofillers with

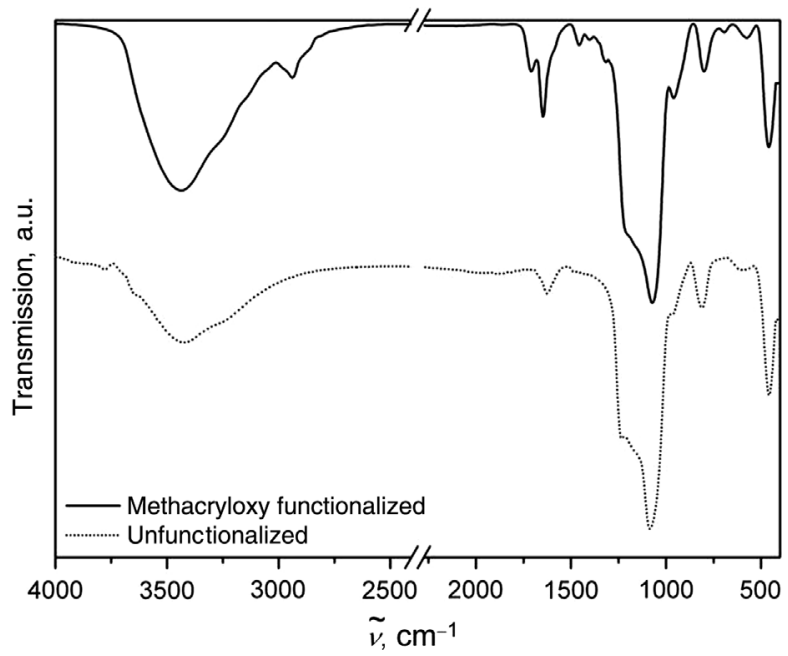

Figure 2 FT-IR spectra of unfunctionalized (bottom) and methacryloxy-modified (top) anisotropic nanoporous silica nanoparticles. 
contents increasing up to $10 \mathrm{wt} \%$. The incorporation of unmodified anisotropic nanoporous filler results in a viscosity considerably increasing with filler content (30.1 Pas at $10 \mathrm{wt} \%, \mathrm{p}<0.05)$. The resulting complex viscosities of uncured nanocomposites with the reference filler Aerosil OX 50 or with modified filler particles lie in the range between 3 and 6 Pas and are comparable to the viscosity of the pure resin (3.4 Pas). Figure 3B shows the results for the hybrid composites with nanofiller contents up to $20 \mathrm{wt} \%$. As shown, the incorporation of Aerosil OX 50 and of the unmodified nanoporous particles both result in a dramatically increased complex viscosity with values up to 200 Pas $(\mathrm{p}<0.05)$. Because of this high increase in viscosity for unmodified nanoporous particles, an incorporation of more than $20 \mathrm{wt} \%$ of these fillers was not possible. However, similar to the case of the nanocomposites, the complex viscosities of hybrid composites with modified nanoporous nanofillers (values between 17 Pas and
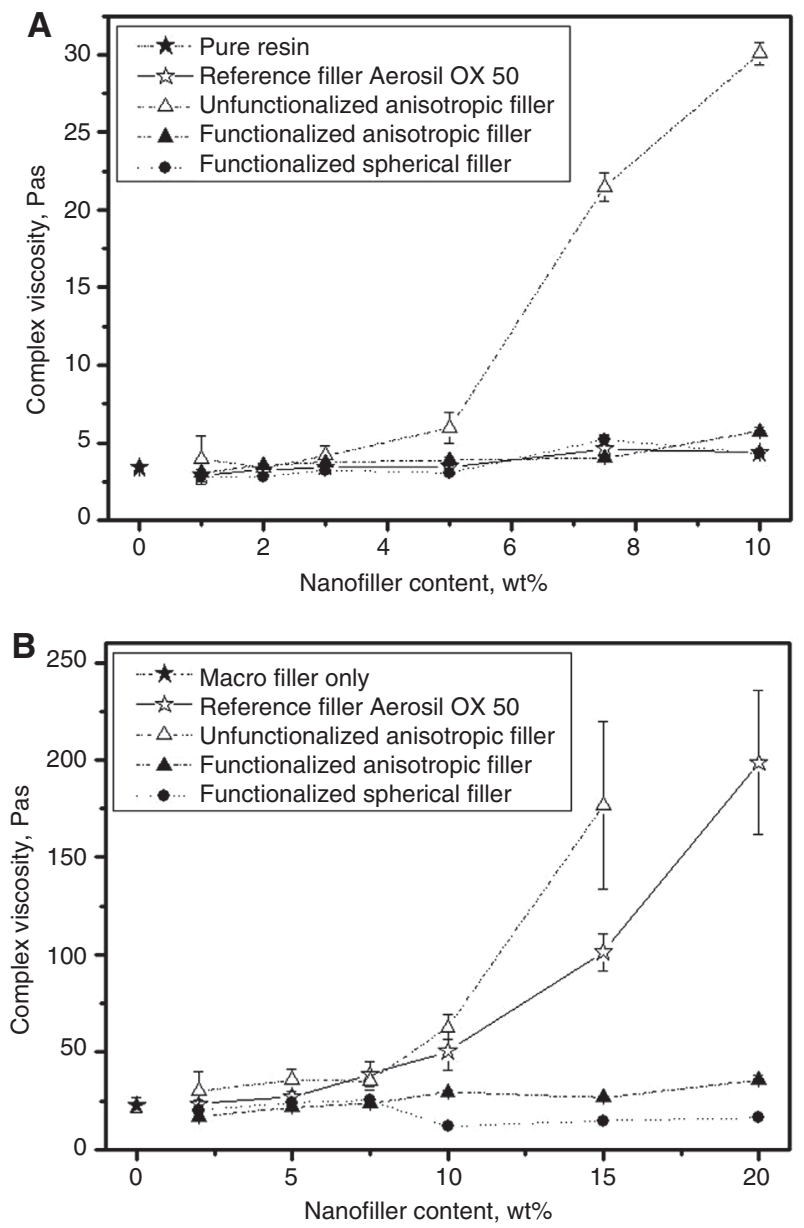

Figure 3 Minimum values of the complex viscosity evaluated with an amplitude sweep between a shear stress of 1 and 1000 Pas at a constant frequency of $1 \mathrm{~Hz}$ for (A) uncured nanocomposites and (B) uncured hybrid composites as a function of nonofiller content.
36 Pas) are in a range comparable to that observed for hybrid composites without any nanofillers (22.8 Pas).

In summary, the viscosities of nanocomposites and hybrid composites with modified filler particles are on a low level, independently of filler load and filler morphology. When Aerosil OX 50 is incorporated into nanocomposites, the influence on viscosity is also rather low because of the reduced amount of hydrophilic silanol groups on the particle surface.

\section{Photopolymerization kinetics and double bond conversion}

Photopolymerization kinetics and double bond conversion were investigated to determine the influence of the unmodified and modified nanoporous nanoparticles on the polymerization process, which in turn influences the mechanical properties. The method to characterize methacrylate-based dental composite materials with NIR spectroscopy was introduced by Stansbury and Dickens [20] and evaluated by Kerbouch et al. [21]

Figure $4 \mathrm{~A}$ shows the vinyl double-bond conversion for the nanocomposites lying in the range between 68 and $80 \%$. For small amounts of unmodified nanoporous nanoparticles in the nanocomposites, the resulting conversion is comparable to that of neat resin. However, incorporation of larger amounts of unmodified anisotropic nanoparticles results in a decreased double-bond conversion (down to $70.0 \%$ for $10 \mathrm{wt} \%$ filler). In contrast, using modified anisotropic nanoporous particles with a hydrophobic methacryloxypropyl modification leads to an increased double bond conversion of approximately $77 \%$ for samples with $1-5 \mathrm{wt} \%(\mathrm{p}<0.05)$ added filler. However, the incorporation of larger amounts of these nanoparticles results in decreasing double-bond conversion ( $71.8 \%$ at $10 \mathrm{wt} \%$ filler).

The results for hybrid composites, as shown in Figure 4B, indicate double-bond conversion between 55 and $75 \%$. While incorporation of unmodified or modified anisotropic nanoporous nanoparticles leads to reduced conversion, the use of modified spherical nanoporous nanoparticles results in stable double-bond conversion rates of approximately $72 \%$. However, improved double-bond conversion upon incorporation of modified nanoporous nanoparticles, as observed for the nanocomposites, was not detected.

\section{Mechanical properties}

Flexural strength $(\sigma)$ and Young's modulus $(E)$ of dental nanocomposites and hybrid composites containing various amounts of unmodified or modified nanoporous 

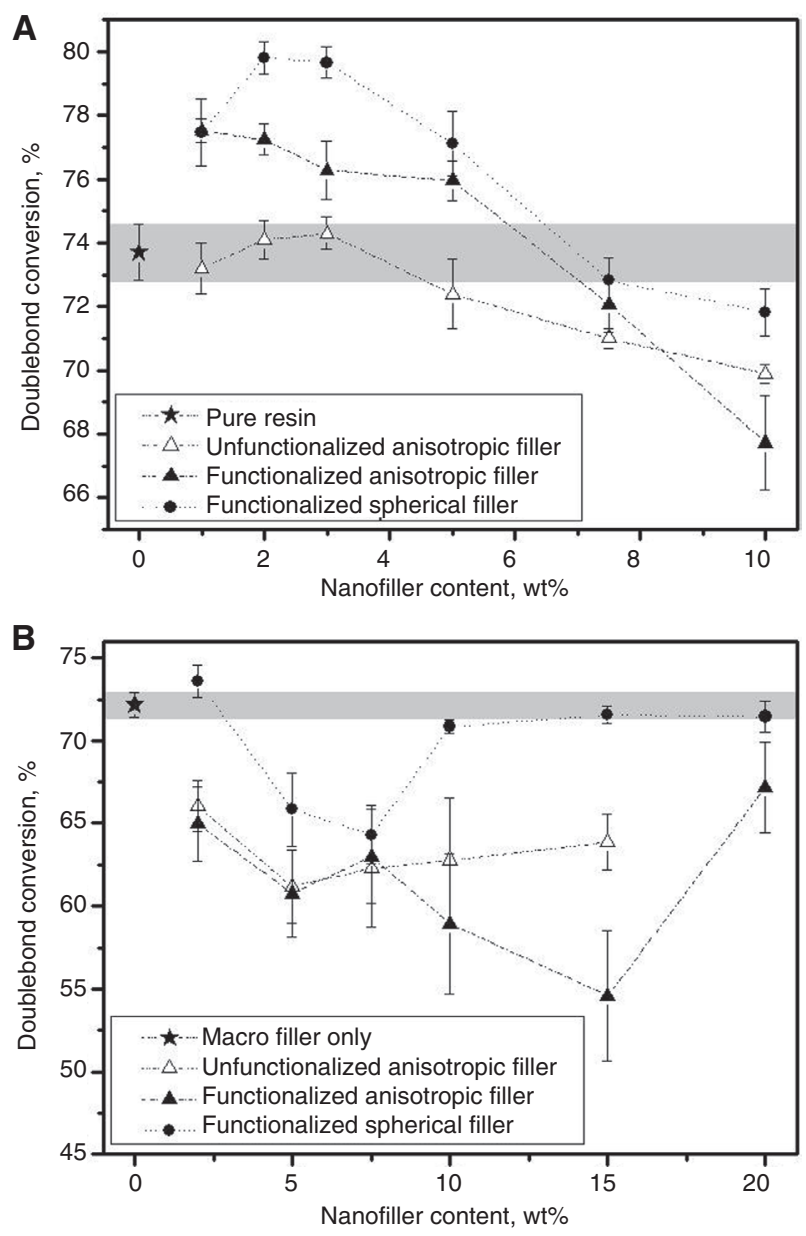

Figure 4 Double bond conversion in the polymerization process of (A) nanocomposites and (B) hybrid composites as a function of nanofiller content.

fillers are shown in Figure 5A-D. Flexural strength represents one of the fundamental characteristics in a critical evaluation of mechanical behavior. As shown in Figure $5 \mathrm{~A}$, flexural strength of nanocomposites increases with increasing content of reference filler up to $103 \mathrm{MPa}$, representing a gain in strength of more than $30 \mathrm{MPa}$ as compared to the pure resin $(68 \mathrm{MPa})$. For nanoporous fillers, a different behavior is observed. The addition of unmodified, anisotropic nanoporous fillers increases the flexural strength, too, but only upon addition of small amounts (up to $3 \mathrm{wt} \%$, resulting in $\sigma=104.1 \mathrm{MPa}$, $\mathrm{p}<0.05$, see Figure $5 \mathrm{~A}$ ). Adding larger amounts results in less favorable properties. By incorporation of modified anisotropic fillers, this effect is retarded up to a filler content of $5 \mathrm{wt} \%$ ( $\sigma=98.5 \mathrm{MPa}, \mathrm{p}<0.05)$. Nevertheless, also in this case a further increase in filler content decreases the flexural strength. However, by the incorporation of modified spherical nanoporous particles, the flexural strength can be positively influenced within the complete range of filler contents studied (up to $10 \mathrm{wt} \%$ ). A maximum value of $\sigma=115.1 \mathrm{MPa}$ is attained.

Figure $5 \mathrm{~B}$ shows the flexural strength of hybrid composites with a total filler content of $50 \mathrm{wt} \%$. In this series up to $20 \mathrm{wt} \%$ filler has been replaced by nanofiller. The unmodified and modified anisotropic nanoporous fillers do not have any influence on the flexural strength of the hybrid composites when compared to a system without any nanofiller $(\sigma=79 \mathrm{MPa})$. However, the incorporation of modified spherical nanoporous particles in the hybrid composites results in reinforcing effects over nearly the complete range of filler contents observed. The maximum strength of 97.2 MPa was determined for a nanofiller content of $2 \mathrm{wt} \%$.

Equally important for the assessment of mechanical behavior of composites is Young's modulus $E$ as a measure for the material's stiffness. As shown in Figure 5C, Young's modulus for nanocomposites strongly increases with nanofiller content. The impregnation of dental resins with the reference filler or with unmodified anisotropic nanoporous particles leads to Young's moduli lying on a relatively low level; in comparison to the neat resin the stiffness increases from $2370 \mathrm{MPa}$ to only $2570 \mathrm{MPa}$ for $10 \mathrm{wt} \%$ of unmodified anisotropic particles. The incorporation of modified anisotropic or spherical particles, however, results in significantly improved stiffness. Both kinds of modified nanoporous particles influence Young's modulus ( $\mathrm{p}<0.05$ ) in a similar way, yielding $E=3089 \mathrm{MPa}$ for $10 \mathrm{wt} \%$ of modified anisotropic fillers and $E=3130 \mathrm{MPa}$ for $10 \mathrm{wt} \%$ of modified spherical fillers. In contrast, as shown in Figure 5D, hybrid composites with $50 \mathrm{wt} \%$ total filler content show a different stiffness behavior both for very low and for increasing nanofiller contents. Unmodified anisotropic fillers do not improve stiffness at all, as has already been shown for nanocomposites in Figure 5C. However, by the incorporation of small amounts of modified nanoporous particles, Young's modulus is improved. Here, in contrast to the corresponding effect in nanocomposites, the morphology of the nanoporous particles has an influence. For example, when regarding hybrid composites with a filler amount of $5 \mathrm{wt} \%$, these show a moderately higher stiffness when impregnated with modified spherical particles ( $E=5720 \mathrm{MPa}$ ) as compared to modified anisotropic particles $(E=5470 \mathrm{MPa})$. However, a further increase in nanofiller content results in significantly decreased stiffnesses.

\section{Dispersion of silica nanoparticles}

Representative SEM images of the synthesized spherical and anisotropic nanoporous nanofillers are depicted in Figure 1A and 1B. The spherical particles shown in Figure 

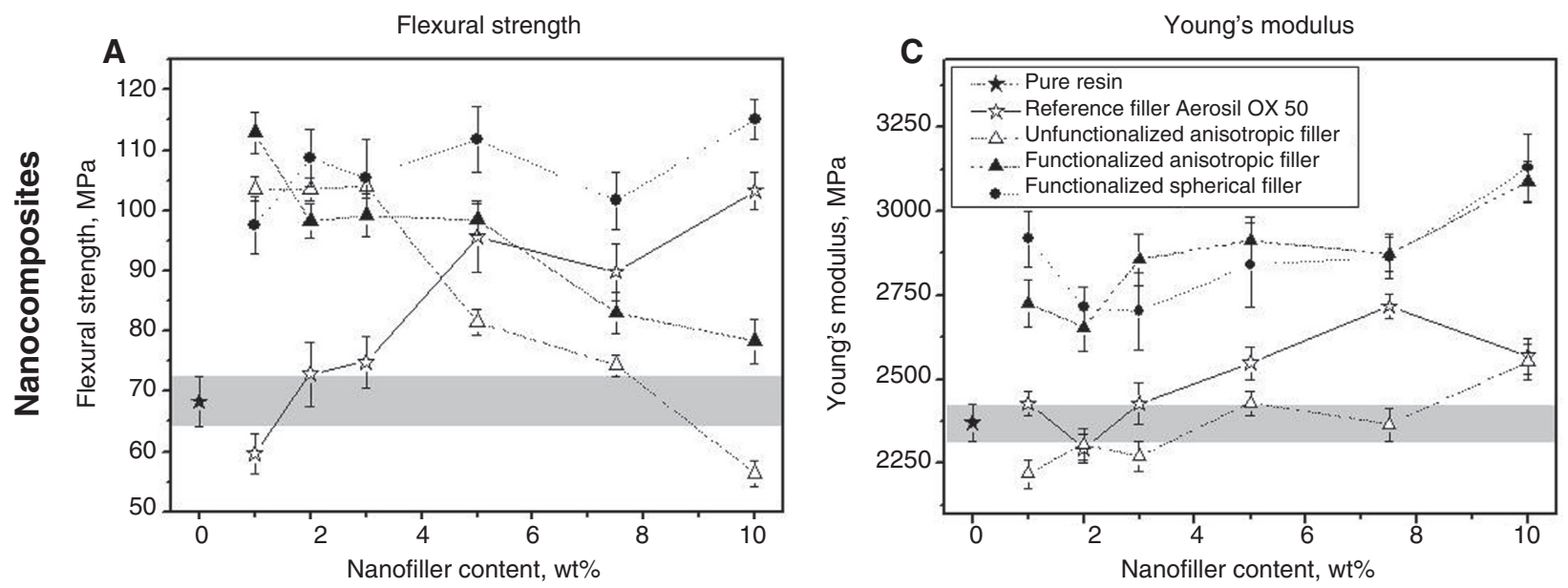

$\mathbf{B}$
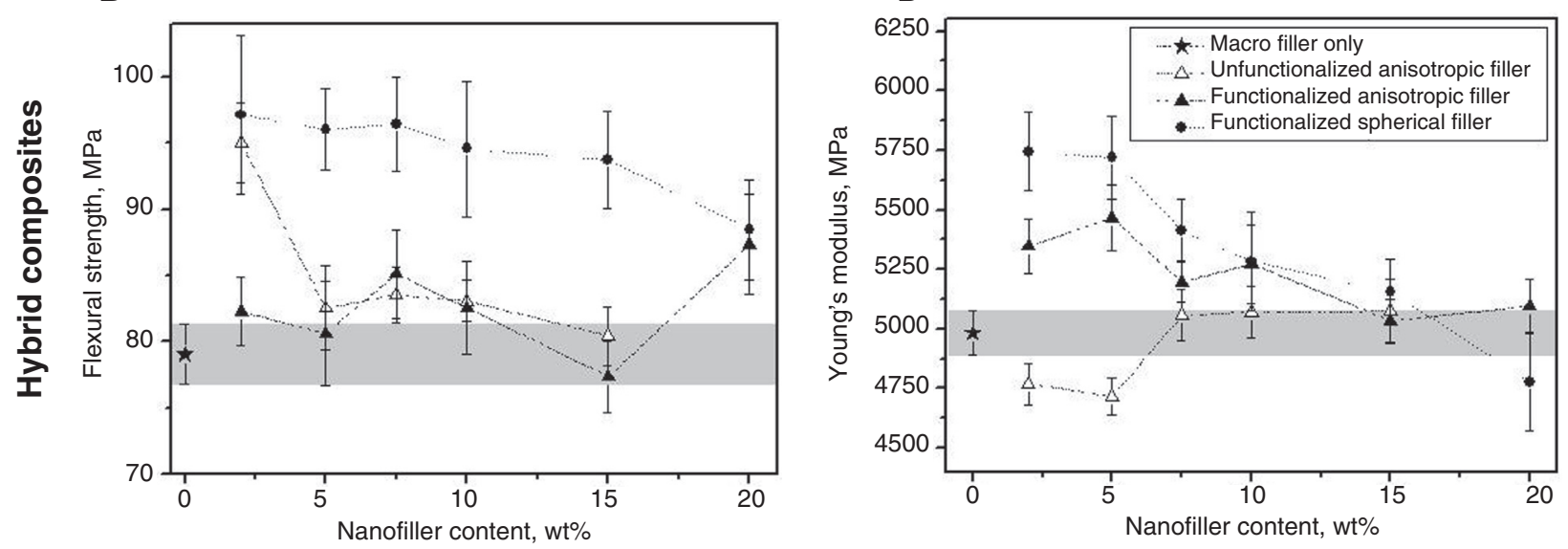

Figure 5 Mechanical properties of nanocomposites (A, C) and hybrid composites (B, D): Flexural strength (A, B) and Young's modulus $(C, D)$ as a function of nanofiller content. The areas shaded in grey correspond to the values observed for the pure resin $(A, C)$ or when only the macro-filler is used $(B, D)$.

1A have an average diameter of $30 \mathrm{~nm}$. The nanoparticles with anisotropic morphology have an average length of several micrometers and an average diameter of 100$150 \mathrm{~nm}$. Representative images of fractured surfaces of the light-cured nanocomposites with $10 \mathrm{wt} \%$ filler nanoparticles are displayed in Figure 6A-D. EDX mappings of two composites are shown in Figure 6E and F: Here, the red color corresponds to silicon, representing the incorporated nanoparticles on the fractured surface, and the black regions represent the organic polymer matrix.

At the fractured surfaces of composites prepared with Aerosil OX 50 particles (Figure 6A), no visible filler agglomerates are detectable. The EDX mapping of this composite (Figure 6E) shows a homogeneous distribution of the particles. The tendency to agglomeration is obviously very small, as expected for a commercially optimized material and in good agreement with the hypothesis that the hydrophobic surface of the Aerosil OX 50 fillers facilitates particle dispersion within the organic matrix.
The SEM image of the composite prepared with modified spherical nanoporous silica particles (Figure 6B) reveals the formation of small agglomerates. The EDX mapping (Figure 6F) shows a high amount of silicon within these agglomerates which indicates that they are formed by the filler particles. The size of these agglomerates amounts to several micrometers with an even distribution. The modified anisotropic nanoporous silica particles seem to have a higher tendency to form agglomerates (Figure 6C). In this case, the agglomerates are of different, sometimes of very large size. The special morphology seems to have a negative effect on the dispersibility within the matrix, but the different size may also play a role. Figure 6D shows the fracture surface of a composite containing unmodified anisotropic silica particles. Very large agglomerates are visible. The hydrophobic modification of the anisotropic particles thus appears to be a crucial step in the composite preparation. Without the attached organic groups, the interactions between the polar silica and the non-polar 
A

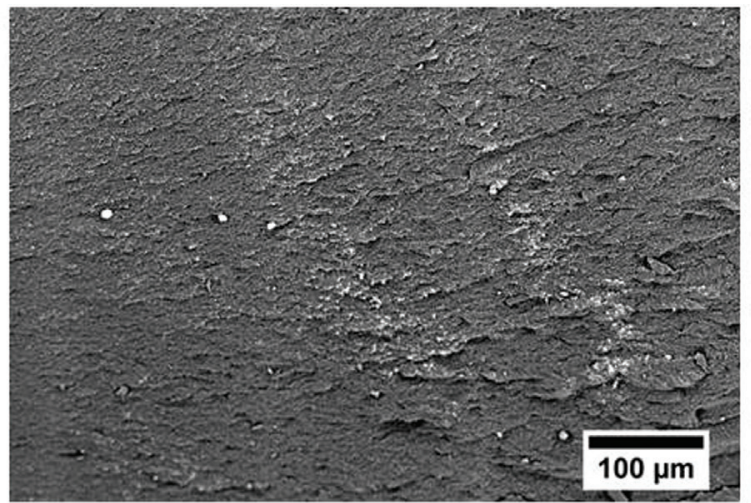

C

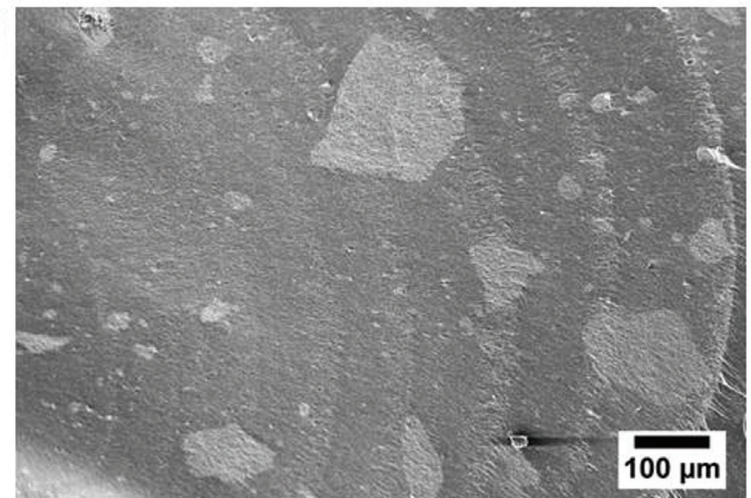

E

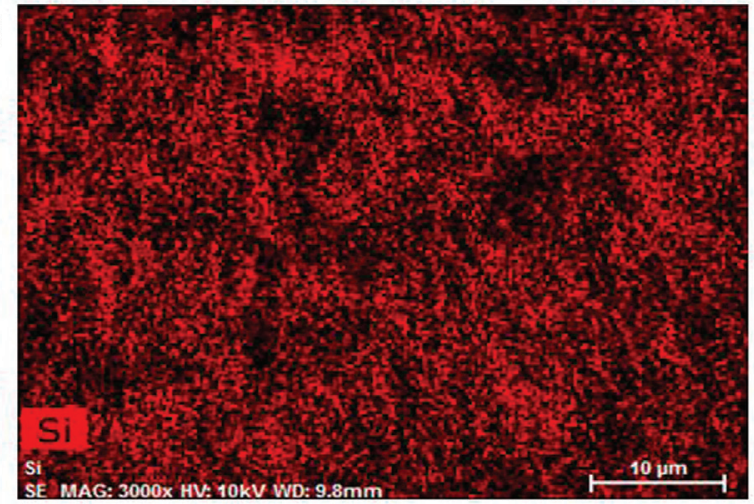

B

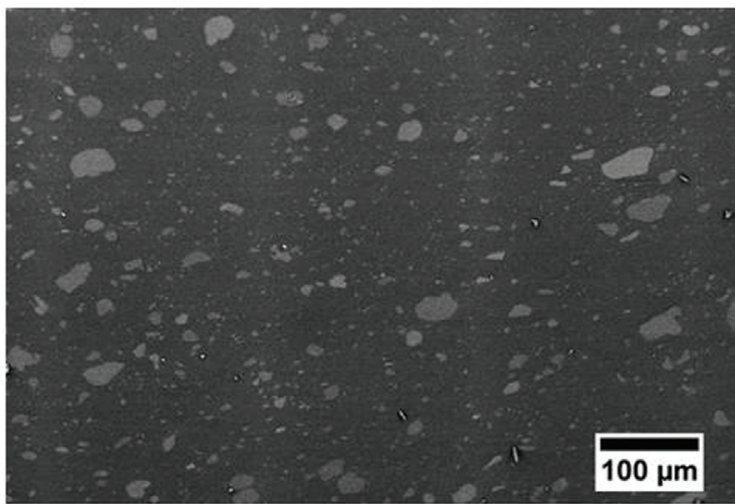

D

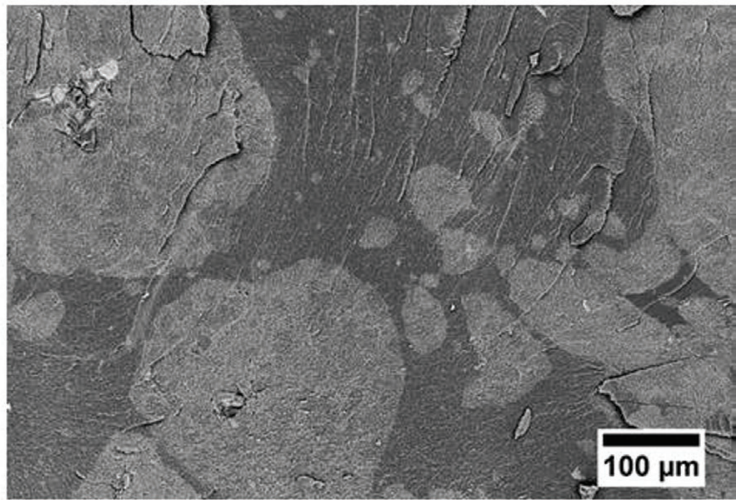

$\mathbf{F}$

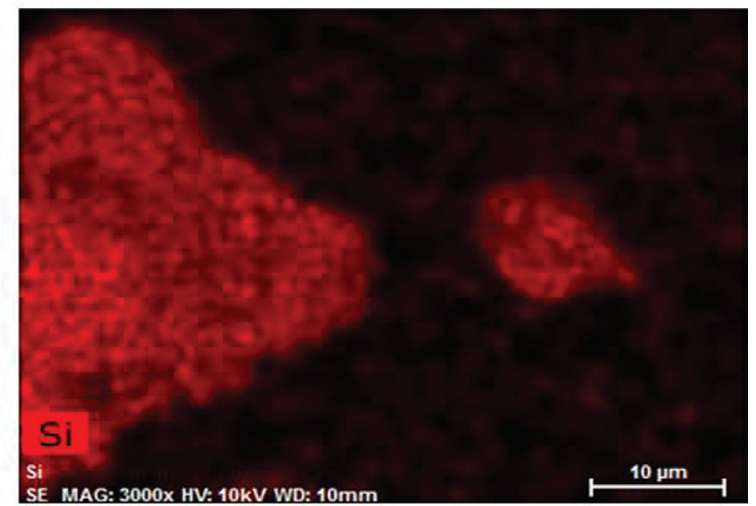

Figure 6 (A-D) SEM images of fracture surfaces of composites prepared with $10 \mathrm{wt} \%$ of (A) Aerosil OX 50, (B) modified nanoporous silica particles with spherical shape, (C) modified nanoporous silica particles with anisotropic shape, (D) unmodified unfunctionalized nanoporous silica particles with anisotropic shape. (E and F) EDX mappings of fracture surfaces of composites prepared with (E) Aerosil OX 50, and (F) modified nanoporous silica particles with spherical shape. Red: Si; black: organic polymer matrix.

matrix are too weak and lead to a strong filler agglomeration causing deteriorated mechanical and physiochemical properties, as described above.

\section{Discussion}

Our objective in this work was to test the possible reinforcement of dental composite materials with specifically synthesized nanoporous nanoparticles, and also to test whether a spherical or anisotropic shape may be of advantage. The incorporation of hydrophilic nanofillers like unmodified anisotropic silica nanoparticles or, to a lesser extent, Aerosil OX 50, leads to an increased viscosity. This results in decreased double-bond conversion because of the lower mobility of the molecules in the composite paste. However, upon incorporation of hydrophobic fillers, like the modified nanoporous fillers, the viscosity remains 
constant over the complete filler content range. Therefore, it could be expected that double bond conversion is not influenced in this case. However, the experiments reveal that the impregnation of the resin with modified fillers result in increased double bond conversion (see Figure 4A) at low filler contents. This effect can be explained by the presence of additional double bonds from the methacrylate groups of the modified filler particles, which are able to participate in cross-linking in a similar way as the monomers of the resin matrix. With further increase of filler content, double bond conversion declines significantly. Two reasons can be given for this behavior: Because of the increased viscosity of some formulations, the methacrylate groups are hindered in their mobility; and due to the increasing aggregation of the nanoparticles, the methacrylate groups bound to their surface are less accessible for the reaction. Both effects decrease the probability of the polymerization reaction taking place.

Not only the double bond conversion, but also the dispersion of the nanoparticles was influenced by high viscosities. Increased nanofiller contents and the resulting high viscosities lead to inhomogeneous particle dispersions in the composite pastes. Generally, the dispersion of nanoparticles was identified by many authors as the main factor influencing mechanical behavior [7-9]. Here, the dispersion of the novel nanoporous nanoparticles seems to influence the mechanical properties, too. Thus, the best mechanical properties are achieved at low nanofiller contents where the dispersion of the nanoparticles is more homogeneous. With increasing nanofiller content, the degree of agglomeration increases and gives rise for a strong decline in flexural strength. However, in contrast to these results observed on nanocomposites with nanoporous fillers, the incorporation of the reference filler Aerosil OX 50 leads to different consequences. As shown in Figure 6A, Aerosil OX 50 is more homogeneously dispersed and, hence, its flexural strength is not negatively influenced by increasing its content. This different behavior can be ascribed to the different manufacturing processes of Aerosil and the nanoporous particles, leading to different surface properties. Aerosil is manufactured by flame pyrolysis and has a smaller number of free silanol groups on the surface than the nanoporous particles which are produced by aqueous sol-gel chemistry. However, at low filler contents, our nanocomposites with incorporated nanoporous particles have a significantly improved flexural strength in comparison to the Aerosil OX 50-filled nanocomposites.

The hypothesis that an anisotropic shape of nanofillers would improve the mechanical properties of composites was not supported by the data obtained. There is no major difference in mechanical properties of composites filled with either nanoporous spherical or nanoporous anisotropic particles which cannot be explained by the different dispersive behavior of both filler types. After modification nanoporous spherical fillers are more effective than the reference system with pyrogenic silica.

\section{Conclusion}

The aim of this investigation was to test whether the mechanical properties of dental composite materials can be improved by the application of nanoporous silica nanoparticles as filling agents. We found that spherical nanoporous particles added in small amounts improve the mechanical properties, which is not the case for the anisotropic nanoporous systems. However, while the difference in morphology of the nanofillers seems to have a minor effect, their surface properties strongly influence their capability of reinforcing the composite. The surface polarity determines the dispersibility of the nanoparticles. A homogeneous dispersion of the nanofillers is a key factor to achieve good reinforcement of the polymeric matrix. Therefore, best results were obtained at low filler contents and with nanoparticles which had been modified by methacryloxypropyl groups which - in addition to supplying polymerizable groups - provide the particles with a more hydrophobic character. This conclusion is also supported by the fact that the viscosity of the precursor mixture remains constant upon the addition of modified nanoparticles, indicating that there is no extensive agglomeration of nanofiller particles in the resin.

\section{Materials and methods}

\section{Materials}

Cetyl trimethylammonium bromide (CTAB), diethanolamine, sodium hydroxide solution, hydrochloric acid, tetraethyl orthosilicate (TEOS), 3-mercaptopropyl trimethoxysilane (MPTES), 3-methacryloxypropyl trimethoxylsilane (MAPTMS), 1,8-diazabicyclo[5.4.0] undec-7-en (DBU), toluene, bis-phenol-A bis-(2-hydroxypropyl)methacrylate (Bis-GMA), tri(ethylene glycol) dimethacrylate (TEGDMA), camphorquinone (CQ) and ethyl-4-dimethylamino-benzoate (EDMAB) were purchased from Sigma-Aldrich Corporation (Germany). A $0.7 \mu \mathrm{m}$ size dental glass filler with $3 \mathrm{wt} \%$ silane (GM 32087) was obtained from Schott Electronic Packaging GmbH (Landshut, Germany), and $40 \mathrm{~nm}$ size silica nanoparticles (Aerosil OX 50) were provided by Evonik Degussa GmbH (Frankfurt, Germany). All chemicals and fillers were used as received. 


\section{Fabrication}

Synthesis of anisotropic nanoporous silica nanoparticles: Nanoporous silica nanoparticles with fiber-like morphology were obtained according to a procedure described in the literature [22]. Two grams of CTAB were dissolved in $1000 \mathrm{~mL}$ of ultrapure water and $9 \mathrm{~mL}$ of $\mathrm{NaOH}(2 \mathrm{M})$ and stirred (at a rotational speed of $250 \mathrm{~min}^{-1}$ ) for $20 \mathrm{~min}$ at $60^{\circ} \mathrm{C}$. Then $13.54 \mathrm{~mL}$ TEOS and $1.14 \mathrm{~mL}$ MPTES were added simultaneously and the mixture was stirred for $2 \mathrm{~h}$ at the same temperature. The solution was vacuum-filtered through a glass frit (porosity 4) and washed with water and ethanol. The white solid product was dried at $60^{\circ} \mathrm{C}$. For calcination, the silica particles were heated at a rate of $1^{\circ} \mathrm{C}$ $\mathrm{min}^{-1}$ to $550^{\circ} \mathrm{C}$ and were kept at this temperature for $5 \mathrm{~h}$ to remove the structure-directing agents.

Synthesis of spherical nanoporous silica nanoparticles: Nanoporous silica nanoparticles with spherical morphology were prepared by dissolving $3.16 \mathrm{~g}$ CTAB in a solution of $75 \mathrm{~mL}$ ultrapure water, $13.36 \mathrm{~mL}$ ethanol and $0.23 \mathrm{~g}$ diethanolamine. The mixture was heated to $40^{\circ} \mathrm{C}$ and stirred. After $30 \mathrm{~min}, 8.56 \mathrm{~mL}$ TEOS were added and stirred for additional $2 \mathrm{~h}$. The solid product was separated from the solution by centrifugation ( $30 \mathrm{~min}$ at $18000 \mathrm{~g}$ ). The particles were washed twice with water and once with ethanol. After drying at $60^{\circ} \mathrm{C}$, the silica particles were calcined as described above [23].

Modification of anisotropic and spherical nanoporous silica nanoparticles: Modification of the silica particles was performed by dispersing $1 \mathrm{~g}$ of nanoporous silica nanoparticles in $40 \mathrm{~mL}$ toluene applying ultrasonic treatment. After the addition of $0.15 \mathrm{~mL} 1,8-\mathrm{diaz}-$ abicyclo[5.4.0]undec-7-en (DBU), the dispersion was heated to $80^{\circ} \mathrm{C}$ under stirring. Then $0.26 \mathrm{~mL}$ MAPTMS were added and the batch was stirred for additional $2 \mathrm{~h}$. The modified silica particles were filtered off using a glass frit (porosity 4) or centrifuged (30 min at $18000 \mathrm{~g}$ ), washed with ethanol and dried at $60^{\circ} \mathrm{C}$.

Preparation of the composites: The organic matrix of the composite materials was prepared by mixing $70 \mathrm{wt} \%$ Bis-GMA and $30 \mathrm{wt} \%$ TEGDMA. CQ (0.5 wt $\%)$ and EDMAB (0.5 wt $\%)$ were added as photoinitiators and accelerator, respectively. To ensure homogeneous dispersions of the nanofillers, they were first dispersed in the pure TEGDMA phase by ultrasonic treatment for $30 \mathrm{~min}$. Subsequently, Bis-GMA, CQ, EDMAB and, for the preparation of hybrid composites, the commercial glass filler (GM32087, Schott) were added and the ultrasonic treatment was continued for another $30 \mathrm{~min}$. For comparison purposes nanocomposites incorporating the reference nanofiller Aerosil OX50 were also prepared with a different dispersion method by using an asymmetric centrifuge (Speedmixer DAC 150 SP, Hauschild, Germany). In this case, all components were mixed for $2 \mathrm{~min}$ at a maximum rotational speed of $3600 \mathrm{~min}^{-1}$ (data shown in the supplementary information).

It is well known that the dispersion of silica nanoparticles has a very strong influence on mechanical properties of dental composites. The ultrasound method we chose to disperse the nanoparticles does not represent the best way for preparing composites with respect to filler distribution. For example, the use of an asymmetric centrifuge gives better dispersion of the nanoparticles and, consequently, improved mechanical properties. As shown in the supplementary material 1, nanocomposites between a filler range from 1 to $10 \mathrm{wt} \%$ exhibit higher flexural strengths when dispersed with an asymmetric centrifuge as compared to nanocomposites dispersed with ultrasound. Since in both cases, though on different levels, the strength shows principally the same dependency on filler content, our reference system, dispersed with the ultrasonic method, is useful for comparison with the described new composite systems. Additionally, the ultrasonic method has the advantage to be less harmful to elongated filler particles than it might be the case for the high shear forces occurring in asymmetric centrifugation or other methods used for dispersion of nanoparticles in polymeric matrices.

\section{Characterization and evaluation}

Scanning electron microscopy: Scanning electron microscopy (SEM) was used to control morphology and size of the nanofillers. Measurements were performed on a field-emission SEM (JSM-6700F SEM, Jeol, Eching, Germany).The manufacturing process of the silica nanoparticles was quality controlled by SEM inspection of the filler particles. The dispersion of the nanoparticles in the organic matrix was also investigated by SEM and by energy dispersive X-ray spectroscopy (EDX) mappings of fractured composite surfaces. For this purpose, a tungsten hairpin filament (JSM-6610LV SEM, Jeol, Eching, Germany) was used. Prior to the measurements, both the fracture surfaces and the pure filler were coated with a thin gold layer to avoid charging effects.

Argon sorption measurements: To characterize the surface areas, pore volumes and pore diameters of the silica nanoparticles, argon sorption measurements were performed at $-186^{\circ} \mathrm{C}$ (Autosorb 1, Quantachrome, Odelzhausen, Germany). Prior to each measurement, the samples were degassed at $200^{\circ} \mathrm{C}$ for $24 \mathrm{~h}$. For calculation of the specific surface area, the Brunauer-Emmett-Teller (BET) method was applied; pore volume and diameter were determined by using the density functional theory (DFT) method. The calculations were performed by means of the software ASiQwin version 2.0 (Quantachrome, Odelzhausen, Germany) choosing "Ar at 87 K_zeolites/silica (cylindrical pores, NLDFT adsorption)" as a calculation model [24].

IR spectroscopy: To characterize the modification of the nanoporous silica nanoparticles, Fourier transformation infrared (FT-IR) spectroscopy was carried out (Tensor 27 instrument, Bruker, Billerica, MA, USA). A small amount of the silica particles was ground with an excess of $\mathrm{KBr}$ and compressed to a pellet by applying a force of $100 \mathrm{kN}$.

Viscosity: The viscosities of uncured hybrid and nanocomposite precursor mixtures were determined by rheological plate/plate measurements (RS 100, Haake, Karlsruhe, Germany) in oscillation mode. Some $0.5 \mathrm{~g}$ of the uncured composite paste was placed between a sliding and a fixed plate separated by a distance of $1 \mathrm{~mm}$. All composites were investigated at a fixed frequency of $1 \mathrm{~Hz}$ and increasing amplitude by using a shear stress of 1-1000 Pa. For comparison purposes, the minimum complex viscosity between 1 and $1000 \mathrm{~Pa}$ was determined. At this point, a predominating viscous behavior was made sure, indicated by the loss modulus exceeding the storage modulus.

Photopolymerization kinetics and double bond conversion: A FT-IR spectrometer (Equinox 55, Bruker, Billerica, MA, USA) equipped with a DTGS detector was used to determine the photopolymerization 
kinetics and vinyl double bond conversion of nano- and hybrid composites by evaluation of absorption spectra in the NIR region (from 6250 to $6100 \mathrm{~cm}^{-1}$ ). The uncured composites were placed in a PTFE ring of $2 \mathrm{~mm}$ width and $12 \mathrm{~mm}$ diameter sealed from both sides by glass slides. Before and after curing by means of a photocuring lamp (Bluephase, Ivoclar Vivadent, Schaan, Liechtenstein; irradiation wavelength 430-490 nm), IR spectra of the composites were taken. According to Kerbouch et al. [21], the conversion of vinyl double bonds can be calculated by:

$$
U=\frac{A_{0}-A_{40 s}}{A_{0}}
$$

where $U$ is the conversion of the methacrylic double bond after irradiation, $A_{0}$ the maximum absorbance of the uncured sample and $A_{40 \mathrm{~s}}$ the maximum absorbance of the composite sample after irradiation, both at $6165 \mathrm{~cm}^{-1}$.

Mechanical properties: Flexural strength and Young's modulus of the cured composites were determined by means of a three-point flexural test according to ISO 4049:2009 [25]. The test specimens were prepared by using $25 \mathrm{~mm} \times 2 \mathrm{~mm} \times 2 \mathrm{~mm}$ PTFE molds. The specimens $(\mathrm{n}=10)$ were photocured for $40 \mathrm{~s}$ on each side and carefully removed from the molds. After storage and postcuring for at least 30 days, the composites were tested with a span between supports of $20 \mathrm{~mm}$ and a crosshead speed of $0.75 \mathrm{~mm} \mathrm{~min}^{-1}$ using a computer-controlled universal mechanical testing machine (type $20 \mathrm{~K}$, UTS Testsysteme, Ulm, Germany) with Phoenix software, version 5.04. The flexural strength was calculated using the following formula:

$$
\sigma=\frac{3 l F}{2 B H^{2}}
$$

where $l$ is the span between supports, $F$ is the failure load, and $B$ and $H$ are the width and the thickness of the test specimen, respectively.

Young's modulus was calculated according to the formula:

$$
E=\frac{F}{D} \frac{l^{3}}{4 B H^{3}}
$$

where $F / D$ is the slope in the linear region of the load displacement curve.

Statistical analysis: Mechanical and physical properties were analyzed by one-way analysis of variance (ANOVA) and post-hoc Bonferroni-Holm tests at a significance level of $\mathrm{p}=0.05$.

Acknowledgments: The authors are thankful to Natalja Wendt and Jann Lippke (Leibniz University of Hannover) for carrying out the sorption measurements and Dr. Britta Hering (Leibniz University of Hannover) for carring out the SEM imaging. This work was supported by the Deutsche Forschungsgemeinschaft as part of the Collaborative Research Center SFB 599. The groups from Hannover Medical School and from Leibniz University of Hannover also profited from support by the research initiative "Biofabrication for NIFE".

\section{References}

1. Ferracane JK. Resin composite - State of the art. Dent Mater 2011;27:29-38.

2. Moszner N, Salz U. New developments of polymeric dental com: posites. Prog Polym Sci 2001;26:535-76.

3. Klapdohr S, Moszner N. New inorganic components for dental filling composites. Monatsh Chem 2005;136:21-45.

4. Rodrigues SA, Scherrer SS, Ferracane JL, Bona AD. Microstruc: tural characterization and fracture behavior of a microhyb rid and a nanofill composite. Dent Mater 2008;24:1281-88.

5. Abell AK, Leinfelder KF, Turner DT. Microscopic observations of the wear of a tooth restorative composite in vivo. J Biomed Mater Res 1983;17:501-7.

6. Zhang L, Gao Y, Chen Q, Tian M, Fong H. Bis-GMA/TEGDMA dental composites reinforced with nano-scaled single crystals of fibrillar silicate. J Mater Sci 2010;45:2521-24.

7. Tian M, Gao Y, Liu Y, Liao Y, Hedin NE, Fong H. Fabrication and. evaluation of Bis-GMA/TEGDMA dental resins/composites containing nano fibrillar silicate. Dent Mater 2008;24:235-43.

8. Chen Q, Zhao Y, Wu W, Xu T, Fong H. Fabrication and evaluation of Bis-GMA/TEGDMA dental resins/composites containing halloysite nanotubes. Dent Mater 2012;28:1071-9.

9. Chen L, Yu Q, Wang Y, Li H. BisGMA/TEGDMA dental composite containing high aspect-ratio hydroxyapatite nanofibers. Dent Mater 2011;27:1187-95.

10. Bégin R, Massé S, Rola-Pleszczynski M, Geoffroy M, Martel $M$, Desmarais $Y$, et al. The lung biological activity of american attapulgite. Environ Res 1987;42:328-39.

11. Perderiset M, Saint Etienne L, Bignon J, Jaurand MC. Interactions of attapulgite (fibrous clay) with human red blood cells. Toxicol Lett 1989;47:303-9.

12. Kresge CT, Leonowicz ME, Roth WJ, Vartuli JC, Beck JC. Ordered mesoporous molecular sieves synthesized by a liquid-crystal template mechanism. Nature 1992;359:710-12.

13. Vallet-Regi M, Rámila A, del Real RP, Pérez-Pariente J. A New Property of MCM-41: Drug Delivery System. Chem Mater 2001;13:308-11.

14. Domingo C, Arcís RW, López-Macipe A, Osorio R, RodríguezClemente R, Murtra J, et al. Dental composites reinforced with hydroxyapatite: Mechanical behavior and absorption/elution characteristics. J Biomed Mater Res 2001;56:297-305.

15. Kim JW, Kim LU, Kim CK. Size control of silica nanoparticles and their surface treatment for fabrication of dental nanocomposites. Biomacromolecules $2007 ; 8: 215-22$.

16. Sing KS, Everett DH, Haul RA, Moscou L, Pierotti RA, Rouquérol J, et al. Reporting physisorption data for gas/solid systems with special reference to the determination of surface area and porosity. Pure Appl Chem 1985;57:603-19.

17. Hesse M, Meier H, Zeeh B. Spektroskopische Methoden in der organischen Chemie, Georg Thieme Verlag: Stuttgart, 2005.

18. Vansant EF, Van Der Voort P, Vrancken KC. Characterisation and Chemical Modifications of Silica Surface, Elsevier: Amsterdam/ Lausanne/New York/Oxford/Shannon/Tokyo, 1995.

19. Klapdohr S, Moszner N. New inorganic components for dental filling composites. Chemical Monthly 2005;136:21-45.

20. Stansbury JW, Dickens SH. Determination of double bond conversion in dental resins by near infrared spectroscopy. Dent Mater 2001;17:71-9. 
21. Kerbouch P, Lebaudy P, Lecamp L, Bunel C. Numerical simulation to correlate photopolymerization kinetics monitoring by RT-NIR spectroscopy and photocalorimetry. Thermochimica Acta 2004;410:73-8.

22. Rambaud F, Vallé K, Thibaud S, Julián-Lopéz B, Sanchez C. Onepot synthesis of functional helicoidal hybrid organic-inorganic nanofibers with periodically organized mesoporosity. Adv Funct Mater 2009;19:215-22.

23. Neumann A, Christel A, Kasper C, Behrens P. BMP2-loaded nanoporous silica nanoparticles promote osteogenic differentiation of human mesenchymal stem cells. RSC Adv 2013;3:24222-30.
24. Tommes M, Cychosz KA. Physical adsorption characterization of nanoporous materials: progress and challenges. Adsorption 2014;202:233-50.

25. International Organization of Standardization (ed.), Standard ISO 4049:2009 Dentistry- Polymer-based restorative materials, International Organization for Standardization, Geneva, 2009.

Supplemental Material: The online version of this article (DOI: 10.1515/bnm-2014-0010) offers supplementary material, available to authorized users. 